Electricity in Poultry Farming

By C. A. Cameron Brown. Pp. $73+12$ plates.

(Oxford: Institute for Research in Agricultural Engineering, 1935.) $2 s$.

Everyone who is interested in poultry farming should read this book. It is written to help the average farm worked by its owner. The author has not in view the large 'luxury farm', worked sometimes altogether by electricity, the true economic value of which it is difficult to estimate. In this book the facts relating to the use of electricity on poultry farms in many different parts of the country are given, together with the work done by research institutes. Lancashire and Yorkshire are very favourably situated, since farmers can buy electricity at $\frac{1}{2}-1 d$. per unit. In districts where the cost is $1 \frac{1}{4} d$. the user has to be economical in its use. All those consulted agree that there is a distinct saving in labour, a gain in cleanliness, safety from fire and exactness of operation by using electricity.

The economies that might be effected by relieving the hen from hatching eggs and brooding chicks and keeping it to its irreplaceable duty of laying eggs has been recognised from the earliest times, particularly by the Egyptians and Chinese. The author describes many types of incubators and gives the relative prices of hot-air machines when worked with oil and with electricity. He states that there is now no question about the technical success of electrical methods in poultry farming. Preston Corporation supplies 300 poultry farms with electricity, the average consumption of each being 7,000 units, and rapid developments are taking place in other areas.

\section{Introduction to Electric Transients}

By Prof. B. Kurtz and Prof. G. F. Corcoran. Pp. $\mathrm{xv}+335$. (New York : John Wiley and Sons, Inc.; London : Chapman and Hall, Ltd., 1935.) 22s. $6 d$. net.

As an introduction to the study of the curious effects produced initially when the switch is closed in an electric circuit due to its inductance and capacitance, we can recommend this book. Each type of transient phenomenon is considered from three angles. The student first analyses the problem by means of the principles and laws of physics. It is then discussed mathematically, and this usually involves solving a differential equation. Finally, the solution is illustrated by actual oscillograms. This experimental verification of sometimes quite unexpected mathematical results will greatly encourage the student and show him that theory is sometimes a great help in practical work.

In addition to showing voltage and current waves, power waves and sound waves obtained experimentally are given. The electrical records of three different tones obtained from the same instrument are very instructive. In this case the relatively pure tone has a fundamental frequency of 466 cycles a second, while the other two composite tones have frequencies of 173 and 87 cycles respectively. A useful chapter is given on Heaviside's operational calculus.
Elementary Textbook of Chemistry: with Laboratory Experiments

By John J. Guenther. Pp. xiv +274. (Now York: The Macmillan Co., 1934.) 7s. 6d. net.

THE contents of this book are quite unlike anything a reader would be led to expect from the indication of the title, as ordinarily understood in Great Britain. The preface explains that the volume is designed mainly to meet the needs of schools of nursing, and the curriculum of these, as required in the United States, and to provide a course for presentation in " 45 hours of time". The ground covered ranges from basic definitions in chemistry and physics, over elementary theory, formulæ, equations and calculations, and the general chemistry, chiefly of the non-metals, to complex organic substances such as higher fatty acid derivatives, aromatics, carbohydrates and foodstuffs, the organic chemistry occupying roughly one-third of the book. Sufficient attention is given to household, hygienic and everyday applications as to provide, virtually, a treatise on domestic science.

The course is intended to be followed rigidly, in the nature of a drill, and copious lecture and practical experiments are detailed. The amount of matter is remarkable, and might provide material for a year's study. Although the needs of the curriculum might be met, it is difficult to see in what respect the cause of scientific education would be furthered by covering the ground in forty-five hours, the distribution of which is left unspecified. An efficient index covers twenty-four pages.

N. M. B.

\section{The Calculus}

By Prof. Hans H. Dalaker and Prof. Henry E. Hartig. Third edition. Pp. viii +276. (New York and London: McGraw-Hill Book Co., Inc., 1935.) 12s. $6 d$. net.

THE application of the calculus to the problems of engineering and physics has now become so extensive that writers on the subject no longer confine their treatment solely to the purely theoretical side. This is very encouraging, and, in the volume under review, the authors have made a very commendable attempt to combine theory and practice without sacrificing the fundamental principles of the calculus. The course covered is fairly comprehensive. Beginning with the ideas of functionality and limits, the student is led on to differentiation, with its manifold applications; integration up to double and triple integrals; infinite series and expansion; and finally, to a brief outline of differential equations. The principles developed are well illustrated by many fully worked-out problems drawn from mechanics, physics and engineering, and the accompanying diagrams are very clearly produced.

For the student, numerous carefully graded exercises, with answers, are provided both for purposes of drill and in illustration of practical problems. A complete and useful summary of formulæ, curves and integrals is given as a final chapter. The whole text is clearly printed and the book should prove very valuable to the advanced practical student. 